\title{
Evaluating apolipoprotein E genotype status and neuroprotective effects against white matter hyperintensity development in high-altitude careers
}

\author{
Richard R. Chapleau ${ }^{1 *}$, CharLee A. Martin', Summer R. Hughes', James C. Baldwin', John Sladky²,3,
} Paul M. Sherman ${ }^{2,4}$ and Michael Grinkemeyer ${ }^{1}$

\begin{abstract}
Objective: This study considers the use of a rapid molecular assay to evaluate apolipoprotein $E(A p o E)$ status in military subjects who have been exposed to high altitude. We hypothesize that ApoE status may be protective against developing brain white matter hyperintensities (WMHs) after high altitude exposure.

Results: We tested 92 subjects who had been exposed to altitudes above 25,000 ft mean sea level, either as pilots or as altitude chamber technicians. We determined subject genetic status using rapid Taqman-style polymerase chain reaction genotyping and evaluated the association of $A p o E$ subtype versus brain lesions using t-tests and two-way analyses of variance. Our results indicate that there is no significant association between ApoE genotype status and the presence of WMHs after high altitude exposure. We did observe a significantly higher number of hours spent at altitude for subjects with the ApoE E2 allele; however, the mechanism by which this may occur is not determined in this study. To more fully elucidate this effect, larger populations would be required to observe greater numbers of subjects with the $E 2$ and $E 4$ alleles.
\end{abstract}

Keywords: Apolipoprotein E, High altitude acclimatization, Neuroprotection, Genotype screening

\section{Introduction}

Genetic variants of the apolipoprotein $E(A p o E)$ gene have been associated with several neurodegenerative disorders including Alzheimer's disease, cognitive impairment, and multiple sclerosis, as well as with varying rates of recovery from traumatic brain injury [1-3]. In the brain, nonneuronal cell types, most notably astroglia and microglia, are the primary producers of ApoE proteins, while neurons preferentially express the receptors for ApoE.

The ApoE4 genotype, specifically, is associated with an increased risk of developing Alzheimer's disease (20\% in controls vs. $90 \%$ in ApoE4 patients) $[4,5]$ and a decreased

\footnotetext{
*Correspondence: richard.chapleau.1@us.af.mil

${ }^{1}$ US Air Force School of Aerospace Medicine, Aeromedical Research Department, Applied Technology and Genomics Division, WrightPatterson AFB, Dayton, OH, US

Full list of author information is available at the end of the article
}

average age of onset from 84 to 68 years, respectively [4]. The mechanism by which $A p o E 4$ participates in disease pathogenesis is not known. Paradoxically, the same genotype is associated with increased neuroprotection against Alzheimer's disease provided by consumption of fish [6].

In a meta-analysis of 42 published studies conducted by Schilling et al. the ApoE4 and ApoE2 genes were associated with increasing burden in magnetic resonance imaging (MRI) markers for both hemorrhagic and ischemic cerebrovascular disease, while ApoE2 correlated with increasing brain white matter hyperintensities (WMHs) [7]. In previous studies from our group [8, 9], increased WMH burden was associated with repetitive nonhypoxic hypobaric exposure, neurologic decompression sickness, and lower neurocognitive test performance as measured on computer-based neurocognitive tests. High altitude aviators are at risk for decompression sickness, 
with an anonymous survey noting a self-reported prevalence of $75.5 \%$ [10].

Because these lesions are destructive to the normal brain architecture, WMHs may be considered a form of traumatic brain injury. In high altitude exposed individuals, these lesions are presumed to be secondary to repetitive hypobaric exposure exacerbated by an increased operation tempo and increased workload during exposure. Determining the etiology of WMHs may lead to important mitigation strategies for high altitude operators. We hypothesized that $A p o E$ status may be a contributing risk factor in developing WMHs after high altitude exposures.

\section{Main text \\ Methods}

\section{Study design and MRI data collection}

We recruited high altitude aviators from both pilot $(\mathrm{n}=44)$ and altitude chamber operator career fields $(\mathrm{n}=48)$. All subjects were male and had brain MRI results obtained from a previous study. All participants were healthy and were selected for the MRI as a consequence of career selection, not as a result of clinical complaint. This study was approved by the Air Force Research Laboratory's Institutional Review Board (FWR20160040H).

\section{Sample collection and laboratory analysis}

We provided consenting subjects with blood tubes and directed them to report to their local medical treatment facility for phlebotomy. Blood samples were sent from the treatment facility to our research laboratory for processing. Nucleic acids were extracted using the Promega Maxwell 16 Blood DNA Purification kit and we obtained ApoE genotype status by performing two polymerase chain reaction genotyping assays (rs7412 and rs429358). Commercially produced Taqman-style assays were purchased directly from Thermo Fisher and the assays were performed on an Applied Biosystems 7500 FAST realtime thermocycler. The two genotyping assays were verified using 10 control samples obtained and pretested at a reference lab.

\section{Statistical analysis}

We evaluated the association between ApoE genotype status and each of the phenotype variables using GraphPad Prism 7.0c. Two-way analysis of variance was performed with genotype as a primary factor and the phenotypes as the other contributing factors. Parameters for the multiple t-tests performed included the assumption of similar scatter and a $10 \%$ false discovery rate using the two-stage step-up method of Benjamini et al. [11]. Descriptive statistics were also obtained in Prism 7, including the mean, median, deviations, and ranges.

\section{Results}

Overall $A p o E$ allele frequencies were consistent with the global allele distribution [12]: $A p o E 2=7.6 \%$, ApoE3 $=78.8 \%$, and $A p o E 4=13.5 \%$. These alleles were spread across four genotypes: ApoE2/ApoE3, ApoE3/ ApoE3, ApoE3/ApoE4, and ApoE4/ApoE4. Performing a two-way analysis of variance revealed that the $A p o E$ genotype status accounted for $0.21 \%$ of the total variance in the population, the interaction between genotype and collected phenotypes accounted for $1.99 \%$, and the differences in the phenotypes themselves accounted for $16.92 \%$ of the variance. The effects of both the interaction and the genotype are not considered significant. Additionally, performing multiple $\mathrm{t}$-tests to evaluate possible associations between genotype and WHM lesion count or volume revealed no significant associations (Table 1).

One interesting result discovered was a positive association between genotype and hours flown above $25,000 \mathrm{ft}$ mean sea level (Table 2). The subjects with the ApoE2/ApoE3 genotype had significantly more hours above this altitude than those with the ApoE3/ApoE3 or

Table 2 Range of accumulated exposures above 25,000 ft mean sea level by genotype

\begin{tabular}{lllrll}
\hline Genotype & Median & Upper & Lower & Mean & Deviation (\%) \\
\hline ApoE2/ApoE3 & 490.5 & 1630 & 21 & 631.4 & 527.5 \\
ApoE3/ApoE3 & 139 & 2000 & 9 & 387.2 & 461.0 \\
ApoE3/ApoE4 & 202 & 1700 & 12 & 405.7 & 455.2 \\
ApoE4/ApoE4 & 665 & 1157 & 173 & 665 & 695.8
\end{tabular}

Table 1 Statistical analysis of multiple t-tests comparing genotype versus WMH lesions

\begin{tabular}{|c|c|c|c|c|c|}
\hline Genotype comparison & $\begin{array}{l}\text { WMH count } \\
\text { p-value }\end{array}$ & $\begin{array}{l}\text { WMH volume } \\
\text { p-value }\end{array}$ & Genotype comparison & $\begin{array}{l}\text { WMH count } \\
\text { p-value }\end{array}$ & $\begin{array}{l}\text { WMH } \\
\text { volume } \\
\text { p-value }\end{array}$ \\
\hline ApoE2/ApoE3 vs. ApoE3/ApoE3 & 0.92 & $>0.99$ & ApoE3/ApoE3 vs. ApoE3/ApoE4 & 0.95 & $>0.99$ \\
\hline ApoE2/ApoE3 vs. ApoE3/ApoE4 & 0.97 & $>0.99$ & ApoE3/ApoE3 vs. ApoE4/ApoE4 & 0.93 & $>0.99$ \\
\hline ApoE2/ApoE3 vs. ApoE4/ApoE4 & 0.97 & $>0.99$ & ApoE3/ApoE4 vs. ApoE4/ApoE4 & 0.95 & $>0.99$ \\
\hline
\end{tabular}


ApoE3/ApoE4 genotypes (p-values of $1.1 \mathrm{e}-9$ and $6.2 \mathrm{e}-6$, respectively). There was no significant association discovered for ApoE2/ApoE3 or ApoE4/ApoE4. However, since there were only two subjects with the latter genotype, no statistical inferences can be made. Looking further into the flight hour details uncovered a wide range of values for the test population. The range of times for the entire population was from 9 to $2000 \mathrm{~h}$.

\section{Discussion}

As more high performance aircraft are being developed with service ceilings greater than $50,000 \mathrm{ft}$, it is becoming increasingly important to understand the impact of altitude on human performance. In addition to high altitude aviators and operators (including military freefall parachutists and aerospace physiologists), the identification of risk factors for altitude-induced illness could influence decisions of adventurers, travelers, and even civilians moving to or living in mountainous regions. Additionally, members of the commercial airline industry may benefit from a greater understanding of the relationship between altitude, disease, and genetics.

Although our hypothesis was disproven by our results, we feel that this study provides critical information in that at least one of the genetic markers related to neurodegeneration is not an apparent risk factor for altitudeinduced brain injury. There may be a protective effect of $A p o E$ status that contributes to a significantly higher number of hours spent at altitude for subjects with the ApoE2 allele; however, the mechanism by which this may occur was not determined in this study. To more fully elucidate this effect, larger populations would be required to observe greater numbers of subjects with the $A p o E 2$ and ApoE4 alleles. Our subject population includes a representative sampling of between $50 \%$ and $90 \%$ of currently qualified high altitude pilots [13], and approximately $1 \%$ of the total population of high altitude pilots ever to have flown. Therefore, our results are expected to highly reflect the true population.

Another alternative to identify if ApoE is implicated in WMH development would be to assess polymorphisms in the gene's promoter region. To that end, we are currently exploring the hypothesis that there is an association between either variant rs405509 (-219G/T) or rs769446 (-427T/C) and WMH development. These variants have been associated in the past with brain functional decline and may be a confounding factor in altitude-induced injury $[14,15]$. We anticipate developing a better understanding of $A p o E$ 's role in altitude-related negative effects through the currently reported results regarding ApoE status and the up-coming work on promoter genotypes.

\section{Limitations}

This study focused on a single gene and had a small sample size with widely varying phenotype data. Additional studies of larger numbers of subjects with high altitude exposure may provide further insight into possible genetic risk factors for altitude-induced neurodegenerative outcomes. Alternative hypothesis testing of additional genes, and/or larger studies with genome-wide technologies, may identify relevant markers.

\section{Abbreviations}

ApoE: apolipoprotein E; MRI: magnetic resonance imaging; WMH: white matter hyperintensity.

\section{Authors' contributions}

RRC conceived idea, developed methods, secured funding, analyzed data, and wrote manuscript. CAM, SRH, and JS performed experiments and edited and approved manuscript. JCB conceived idea and edited and approved manuscript. PS conceived idea, performed experiments, and edited and approved manuscript. MG conceived idea, secured funding, analyzed data, and edited and approved manuscript. All authors read and approved the final manuscript.

\section{Author details}

${ }^{1}$ US Air Force School of Aerospace Medicine, Aeromedical Research Department, Applied Technology and Genomics Division, Wright-Patterson AFB, Dayton, OH, US. ${ }^{2}$ US Air Force School of Aerospace Medicine, Aeromedical Research Department, Operational Health and Performance Research Division, Wright-Patterson AFB, Dayton, OH, US. ${ }^{3}$ 59th Medical Wing, Department of Neurology, Joint Base San Antonio-Lackland, Houston, TX, US. ${ }^{4}$ 59th Medical Wing, Department of Neuroradiology, Joint Base San Antonio-Lackland, Houston, TX, US.

\section{Acknowledgements}

The authors would like to express their sincere thanks to the MRI and logistics teams at Joint Base San Antonio and Wright-Patterson Air Force Base: Paul Vu, Michael Lang, Angela Palitto, Owen Scott, Hope Soliz, Pedro Ramos, Ellie Vasquez, and Tracy Oumeddour. The views expressed in this article are those of the authors and do not necessarily reflect the official policy or position of the Air Force, the Department of Defense, or the US Government.

\section{Competing interests}

The authors are employees of or under contract to the United States Air Force.

\section{Availability of data and materials}

Summary data from this study, including genotype and hyperintensity measurements, are available from the corresponding author upon reasonable request.

\section{Consent for publication}

Not applicable.

\section{Ethics approval and consent to participate}

This study was approved by the Air Force Research Laboratory's Institutional Review Board (FWR20160040H). All subjects were individually contacted and consented into the study prior to providing samples for genetic testing and association testing with MRI results.

\section{Funding}

This work was funded internally by the United States Air Force and the Defense Health Agency. The funding body had neither direct nor indirect influence on the publication of this study.

\section{Publisher's Note}

Springer Nature remains neutral with regard to jurisdictional claims in published maps and institutional affiliations. 
Received: 15 September 2018 Accepted: 17 October 2018

Published online: 25 October 2018

\section{References}

1. Friedman G, Froom P, Sazbon L, Grinblatt I, Shochina M, Tsenter J, Babaey S, Yehuda B, Groswasser Z. Apolipoprotein E-epsilon4 genotype predicts a poor outcome in survivors of traumatic brain injury. Neurology. 1999;52(2):244-8.

2. Mayeux R, Ottman R, Maestre G, Ngai C, Tang MX, Ginsberg H, Chun M, Tycko B, Shelanski M. Synergistic effects of traumatic head injury and apolipoprotein-epsilon 4 in patients with Alzheimer's disease. Neurology. 1995;45(3 Pt 1):555-7.

3. Sorbi S, Nacmias B, Piacentini S, Repice A, Latorraca S, Forleo P, Amaducci L. ApoE as a prognostic factor for post-traumatic coma. Nat Med. 1995;1(9):852.

4. Corder EH, Saunders AM, Strittmatter WJ, Schmechel DE, Gaskell PC, Small GW, Roses AD, Haines JL, Pericak-Vance MA. Gene dose of apolipoprotein E type 4 allele and the risk of Alzheimer's disease in late onset families. Science. 1993;261(5123):921-3.

5. Spinney L. Alzheimer's disease: the forgetting gene. Nature. 2014;510(7503):26-8.

6. Morris MC, Brockman J, Schneider JA, Wang Y, Bennett DA, Tangney CC, van de Rest $\mathrm{O}$. Association of seafood consumption, brain mercury level, and $\mathrm{APOE} \varepsilon 4$ status with brain neuropathology in older adults. JAMA. 2016;315(5):489-97.

7. Schilling S, DeStefano AL, Sachdev PS, Choi SH, Mather KA, DeCarli CD, Wen W, Høgh P, Raz N, Au R, et al. APOE genotype and MRI markers of cerebrovascular disease: systematic review and meta-analysis. Neurology. 2013;81(3):292-300.

8. McGuire SA, Sherman PM, Brown AC, Robinson AY, Tate DF, Fox PT, Kochunov PV. Hyperintense white matter lesions in 50 high-altitude pilots with neurologic decompression sickness. Aviat Space Environ Med. 2012;83(12):1117-22.

9. McGuire S, Sherman P, Profenna L, Grogan P, Sladky J, Brown A, Robinson A, Rowland L, Hong E, Patel B, et al. White matter hyperintensities on MRI in high-altitude U-2 pilots. Neurology. 2013;81(8):729-35.

10. Bendrick GA, Ainscough MJ, Pilmanis AA, Bisson RU. Prevalence of decompression sickness among U-2 pilots. Aviat Space Environ Med. 1996;67(3):199-206.

11. Benjamini Y, Krieger AM, Yekutieli D. Adaptive linear step-up procedures that control the false discovery rate. Biometrika. 2006;93(3):491-507.

12. Genomes Project Consortium, Auton A, Brooks LD, Durbin RM, Garrison EP, et al. A global reference for human genetic variation. Nature. 2015;526(7571):68-74.

13. Bettancourt M. Killer at $70,000 \mathrm{ft}$ : the occupational hazards of flying the U-2. Air and Space/Smithsonian May 2012 Web. 28 September 2018.

14. Ma C, Zhang Y, Li X, Zhang J, Chen K, Liang Y, Chen Y, Liu Z, Zhang Z. Is there a significant interaction between APOE rs405509 T/T, and e4 genotypes on cognitive impairment and gray matter volume? Eur J Neurol. 2016;23(9):1415-25.

15. Limon-Sztencel A, Lipska-Zietkiewicz BS, Chmara M, Wasag B, Bidzan L, Godlewska BR, Limon J. The algorithm for Alzheimer risk assessment based on APOE promoter polymorphisms. Alzheimers Res Ther. 2016;8:19.
Ready to submit your research? Choose BMC and benefit from:

- fast, convenient online submission

- thorough peer review by experienced researchers in your field

- rapid publication on acceptance

- support for research data, including large and complex data types

- gold Open Access which fosters wider collaboration and increased citations

- maximum visibility for your research: over 100M website views per year

At BMC, research is always in progress.

Learn more biomedcentral.com/submissions 